\title{
Induction of ferroptosis by ATF3 elevation alleviates cisplatin resistance in gastric cancer by restraining Nrf2/Keap1/xCT signaling
}

\author{
Dazhi Fu ${ }^{{ }^{*}}$, Chunxiao Wang ${ }^{2}$, Lei Yu ${ }^{1}$ and Rui Yu ${ }^{1}$
}

\author{
${ }^{*}$ Correspondence: \\ fudazhihospital@yandex.com \\ 1 Department of General \\ Surgery, First Affiliated \\ Hospital of China Medical \\ University, Liaoning Province \\ Shenyang City Heping \\ District Nanjingbei Road 155, \\ Shenyang 110001, Liaoning, \\ People's Republic of China \\ Full list of author information \\ is available at the end of the \\ article
}

\begin{abstract}
Background: Currently, resistance against cisplatin (DDP) is a frequent problem for the success of advanced gastric carcinoma (GC) chemotherapy. Here, we sought to investigate the function of activating transcription factor 3 (ATF3) $n$ GC chemoresistance.
\end{abstract}

Methods: Expression of ATF3 was determined in GC cell lines (MNK45, SGC7901, and BGC823) and cisplatin (DDP)-resistant cells (SGC7901/DDP and BGC823/DDP). Biological informatics was performed to analyze ATF3 expression and prognosis in GC patients. Cisplatin resistance was evaluated. Ferroptosis was detected after ATF3 transfection of cells. The underlying molecular mechanism was also investigated.

Results: Transcripts of ATF3 were decreased in GC cells and GC tissues. Kaplan-Meier plotter analysis revealed that ATF3 expression was positively related to the overall survival of GC patients. In particular, lower levels of ATF3 were observed in cisplatinresistant SGC7901/DDP and BGC823/DDP relative to their parental cells. Notably, ATF3 elevation sensitized cisplatin-resistant cells to cisplatin. Mechanically, compared with parental cells, SGC7901/DDP and BGC823/DDP cells exhibited lower ferroptosis evident by lower ROS, MDA and lipid peroxidation and higher intracellular GSH levels. However, ATF3 elevated ferroptosis in SGC7901/DDP and BGC823/DDP cells. Intriguingly, ATF3 overexpression together with ferroptosis activator erastin or RSL3 treatment further enhanced ferroptosis and cisplatin resistance; however, the ferroptosis suppressor liproxstatin-1 reversed the function of ATF3 in ferroptosis and cisplatin resistance. Additionally, cisplatin-resistant cells exhibited stronger activation of Nrf2/Keap1/xCT signaling relative to parental cells, which was restrained by ATF3 up-regulation. Importantly, restoring Nrf2 signaling overturned ATF3-mediated ferroptosis and cisplatin resistance.

Conclusion: ATF3 may sensitize GC cells to cisplatin by induction of ferroptosis via blocking Nrf2/Keap1/xCT signaling, supporting a promising therapeutic approach for overcoming chemoresistance in GC.

Keywords: Gastric cancer, ATF3, Cisplatin resistance, Ferroptosis, Nrf2/Keap1 signaling included in the article's Creative Commons licence and your intended use is not permitted by statutory regulation or exceeds the permitted use, you will need to obtain permission directly from the copyright holder. To view a copy of this licence, visit http://creativecommons. org/licenses/by/4.0/. 


\section{Background}

Gastric cancer (GC) currently ranks as the fifth most prevalent malignancy and the third in cancer-related mortality in modern society [1,2]. Globally, GC poses a considerable health threat because epidemiological investigation confirms over 1 million estimated new cases of GC and almost 800,000 deaths annually [3]. Over 70\% of GC cases occur in developing countries including China [4]. Currently, traditional radical surgery and palliative chemotherapy remain the mainstay of GC treatment. Cisplatin (DDP)-based chemotherapy is deemed as a common first-line treatment for cancer patients including GC. Nevertheless, GC patients with advanced disease usually acquire resistance to chemotherapy, resulting in a somber prognosis with a median overall survival of 8-11 months [5].

Cell death is crucial for the prevention of hyperproliferative diseases including cancer. Increasing cancer cell sensitivity to chemotherapy usually originates from cancer cell death. Ferroptosis was coined in 2012 and was recently defined as a new form of cell death characterized by iron-dependent lipid peroxidation [6]. Interestingly, reactive oxygen species (ROS) and malondialdehyde (MDA) are fairly specific lipid peroxidation products and frequently defined as markers of ferroptosis. Ferroptosis can be activated in specific pathological states and its maladjustment has been implicated in several physiological and pathological processes, such as neurodegeneration, renal failure and lung injury $[7,8]$. Of interest, cancer cells can adapt to an oxidative environment to control ferroptosis that may reshape the tumor niche to facilitate tumor growth and progression. For instance, inhibition of ferroptosis by activating nuclear factor (erythroid-derived)like 2 (Nrf2) signaling facilitates brain tumor growth [9]. Importantly, emerging evidence has implicated ferroptosis in chemoresistance in several cancers [10-13]. In head and neck carcinoma, cystine deficiency induces ferroptosis evident by glutathione (GSH) depletion and lipid ROS accumulation and thereby overcomes cisplatin resistance [10]. Moreover, induction of ferroptosis by ginkgetin enhances the therapeutic efficacy of cisplatin in non-small-cell lung cancer [12]. Therefore, investigating the crosstalk between ferroptosis and chemoresistance has provided a new insight for tumor intervention.

Activation transcription factor 3 (ATF3) is a common member of the ATF/ cAMP response element-binding (CREB) protein family. As a stress-induced transcription factor, ATF3 is increased in obese mice, and targeting ATF3 exhibits suppressive efficacy towards lipid accumulation $[14,15]$. Recently, ATF3 has become a subject of interest in carcinoma due to its frequent and aberrant expression in multiple cancers. However, according to existing investigations, the function of ATF3 is complicated and contradictory in carcinogenesis. For one thing, ATF3 can act as an oncogene to regulate the development of breast cancer and skin cancer $[16,17]$. On the other hand, several studies substantiate the down-regulation of ATF3 in cancers and support it as a suppressor in oncogenesis $[18,19]$. Additionally, ATF3 is responsible for chemoresistance in non-small cell lung carcinoma [20] and nasopharyngeal cancer [21]. In the current study, we sought to investigate the function of ATF3 in cisplatin resistance in GC cells. Notably, a recent study confirmed that ATF3 promotes ferroptosis in fibrosarcoma cells [22]. Therefore, we also investigated whether ferroptosis is involved in ATF3 function in GC chemoresistance and how ATF3 regulates ferroptosis during this process. Our findings suggested that ATF3 could sensitize GC cells to cisplatin by ferroptosis induction via blocking 
Nrf2/Keap1/xCT signaling. Thus, the present research may implicate ATF3 as a promising therapeutic candidate to overcome chemoresistance in GC.

\section{Materials and methods}

\section{Cell lines and culture}

Human gastric epithelial cells (GES-1) (No. BNCC353464) were obtained from the Beina Chuanglian Biotechnology Research Institute (Beijing, China). Human GC cell lines (MNK45, SGC7901 and BGC823) were bought from the ATCC (Rockville, MD, USA). All cells were kept in RPMI 1640 medium (HyClone, South Logan, UT, USA) supplemented with $10 \%$ fetal calf serum (FCS), $1 \%$ penicillin and streptomycin. For the generation of cisplatin (DDP)-resistant SGC7901 and BGC823 cells, the parental GC cells were treated with gradually increased concentrations of cisplatin as in previous reports [23]. Briefly, the parental SGC7901 and BGC823 cells in the log-growth phase were incubated in culture medium containing cisplatin $(0.1 \mu \mathrm{g} / \mathrm{ml}$ final concentration). Fourteen days later, the cisplatin dose was gradually increased to $0.2 \mu \mathrm{g} / \mathrm{ml}$ for further incubation for 2 weeks. Then, cisplatin concentration was gradually elevated to $0.4 \mu \mathrm{g} / \mathrm{ml}$ and $0.8 \mu \mathrm{g} /$ $\mathrm{ml}$ according to similar procedures until cells could maintain stable growth and repeated passage. The final prepared cells were cisplatin-resistant cells and defined as SGC7901/ DDP and BGC823/DDP, respectively. For culture, all cells were maintained at $37^{\circ} \mathrm{C}$ in a humidified incubator with $5 \% \mathrm{CO}_{2}$ and $95 \% \mathrm{O}_{2}$.

\section{Cell treatment}

The GC cell lines (SGC7901, BGC823, SGC7901/DDP, BGC823/DDP) were stimulated with the indicated doses of cisplatin. Furthermore, the SGC7901/DDP and BGC823/ DDP cells were treated with ferroptosis agonist erastin $(0.8 \mu \mathrm{M})$, RSL3 $(0.1 \mu \mathrm{M})$ or antagonist liproxstatin-1 ( $80 \mathrm{nM}$, both from Selleck, Houston, Texas, USA) before and during cisplatin exposure [11].

\section{Construction of expression plasmids}

To generate the expressed plasmids, total RNA from GC cells was extracted using the TRIzol reagent (Invitrogen, Carlsbad, CA, USA), followed by the synthesis of the firststrand cDNA with the SuperScript II First Strand Synthesis System (Invitrogen). Then, the full-length ATF3 and Nrf2 cDNA was PCR amplified. Then, the prepared cDNA was digested with the restriction enzymes and cloned into pcDNA3.1(+) plasmids to obtain the recombinant pcDNA-ATF3 and pcDNA-Nrf2 vectors. Subsequently, cisplatinresistant GC cells were seeded in 24-well plates and transfected with pcDNA-ATF3 and pcDNA-Nrf2 plasmids using Lipofectamine 2000 (Invitrogen). During this process, cells transfected with empty vectors were defined as the negative control. Approximately $48 \mathrm{~h}$ later, western blotting was conducted to evaluate the transfected efficacy of plasmids.

\section{Human Protein Atlas and Kaplan-Meier plotter assay}

The protein expression of ATF3 in normal gastric tissue and GC tissues was analyzed online using the Human Protein Atlas (https://www.proteinatlas.org/). The correlation between ATF3 and survival rate in GC patients was evaluated online using the Kaplan-Meier plotter (http://kmplot.com/analysis/index.php). The Kaplan-Meier 
plotter database is an online system to analyze mRNA Affymetrix Genechip and RNA-sequencing datasets for GC patients. All information was obtained from the online database and not collected by ourselves.

\section{Quantitative RT-PCR (qRT-PCR)}

After total RNA extraction and cDNA synthesis, real-time PCR was conducted using the SYBR Premix Ex Taq II Kit (TaKaRa, Dalian, China) to quantify the transcription of ATF3. The specific oligonucleotide primer sequences for ATF3 and GAPDH are shown in Table 1 and were bought from Shanghai Sangon Co., Ltd (Shanghai, China). The cycling parameters were as follows: $95{ }^{\circ} \mathrm{C} 10 \mathrm{~min}$ for initial denaturation; $94{ }^{\circ} \mathrm{C}$ or $15 \mathrm{~s}$, $58^{\circ} \mathrm{C}$ for $30 \mathrm{~s}$, and $72{ }^{\circ} \mathrm{C}$ for $15 \mathrm{~s}$ for 40 cycles. All specimens were subjected to the ABI PRISM 7000 system (Applied Biosystems; Foster City, CA, USA) for PCR reaction. The expression of each gene was analyzed by the $2^{-\Delta \Delta \mathrm{Ct}}$ equation. The quantification of target gene expression was conducted by introducing the internal control GAPDH.

\section{Cell viability evaluation}

Parental GC cells and cisplatin-resistant SGC7901/DDP and BGC823/DDP cells treated with pcDNA-ATF3, pcDNA-Nrf2, erastin, RSL3 or liproxstatin-1 were incubated with cisplatin for $48 \mathrm{~h}$. Then, $10 \mu \mathrm{l}$ of Cell Counting Kit (CCK)-8 solution (Nanjing Jiancheng Bioengineering Institute, Nanjing, China) was supplemented into each well for $3 \mathrm{~h}$. Afterwards, cell viability was assessed by capturing the absorbance at $450 \mathrm{~nm}$ using a spectrophotometer.

\section{Immunoblot assay}

Cells were subjected to the indicated treatment and then lysed at $4{ }^{\circ} \mathrm{C}$ in a RIPA lysis buffer. After protein quantification by the BCA protein assay kit (Beyotime, Shanghai, China), $30 \mu \mathrm{g}$ of protein was resolved by $12 \%$ SDS-PAGE and transferred electrophoretically to a PVDF membrane. Subsequently, membranes were hybridized with primary antibodies against human ATF3 (1:1000), Nrf2 (1:800), Keap1 (1:1000), and xCT (1:8000) (all from Abcam, Cambridge, UK, USA) overnight at $4{ }^{\circ} \mathrm{C}$. After incubation with horseradish peroxidase-conjugated secondary antibody, the ECL reagent (Beyotime) was added to visualize the binding signals. Densitometric analysis of immunostained protein bands was conducted using a Gel Doc XR imaging system (Bio-Rad Laboratories, Hercules, CA, USA) and Image J software (National Institutes of Health).

Table 1 Primer sequences of qRT-PCR

\begin{tabular}{llc}
\hline Name & Primer sequences $\left(\mathbf{5}^{\prime}-\mathbf{3}^{\prime}\right)$ & $\begin{array}{c}\text { Product } \\
\text { size (bp) }\end{array}$ \\
\hline ATF3 & Sense, 5'-CTGGAAAGTGTGAATGCTGAAC-3' \\
Anti-sense, 5'-ATTCTGAGCCCGGACAATAC-3' & 117 \\
GAPDH & Sense, 5'-CAAGAGCACAAGAGGAAGAGAG-3' \\
& Anti-sense, 5'-CTACATGGCAACTGTGAGGAG-3' & 102 \\
\hline
\end{tabular}




\section{Cell death assay}

Cell death evaluation was performed with the previously described Annexin V-FITC/ PI staining method [24]. Briefly, cells under various treatments were collected and resuspended in $500 \mu \mathrm{l}$ of Annexin V Binding buffer (Beyotime). Then, cells were further incubated with $10 \mu \mathrm{l}$ of Annexin V-FITC and $5 \mu \mathrm{l}$ of PI in the dark. The positively stained cells were then counted using a FACSAria II flow cytometer (BD Biosciences, USA) with Cell Quest software.

\section{Reactive oxygen species (ROS) measurement}

Cellular ROS generation was measured by adding $20 \mu \mathrm{M}$ of cell-permeating probe $2^{\prime}, 7^{\prime}$-dichlorodihydrofluorescein diacetate (DCFH-DA; Sigma). After incubation for $0.5 \mathrm{~h}$, the produced fluorescence intensity was determined using a spectrofluorimeter at $485 \mathrm{~nm}$ excitation wavelength and $530 \mathrm{~nm}$ emission wavelength. All protocols were performed following the product instructions.

\section{Malondialdehyde (MDA) and glutathione (GSH) assay}

The contents of MDA in cells were determined using a commercial MDA Detection Kit (Nanjing Jiancheng Bioengineering Institute). Briefly, cells that received various treatments were lysed to prepare supernatants. After that, the supernatants were reacted with $200 \mu \mathrm{l}$ of MDA reaction solution at $100^{\circ} \mathrm{C}$ for $15 \mathrm{~min}$. Then, MDA levels were measured at $532 \mathrm{~nm}$ using a microplate reader. For cellular GSH detection, a commercial GSH Detection Kit (Nanjing Jiancheng Bioengineering Institute) was introduced. The absorbance at $420 \mathrm{~nm}$ was captured to calculate cellular GSH contents. All procedures were conducted according to instructions provided by manufacturers.

\section{Evaluation of lipid peroxidation}

For intracellular lipid peroxidation assay, cells were collected and resuspended in PBS buffer. Then, cells were stained with $2.5 \mu \mathrm{M}$ BODIPY-C11 fluorescent dye (Invitrogen) for $10 \mathrm{~min}$ in a cell culture incubator. After rinsing with PBS three times, all specimens were analyzed using a flow cytometer (BD Biosciences).

\section{Statistical analysis}

Statistical calculations were carried out using SPSS 22.0 software. Data are shown as mean \pm standard deviation (SD). The data shown in the figure represented at least three independent experiments. The significance of differences was analyzed using Student's $t$-test for two experimental groups and ANOVA with the Student-Newman-Keuls test for three or more groups. A $p$-value $<0.05$ was defined as statistically significant $\left({ }^{*} P<0.05,{ }^{* *} P<0.01\right)$.

\section{Results}

\section{Expression and prognosis of ATF3 in gastric cancer cells and tissues}

To elucidate the function of ATF3 in GC, we first determined the expression of ATF3 in GC cells. As shown in Fig. 1A, in contrast to the human gastric epithelial 

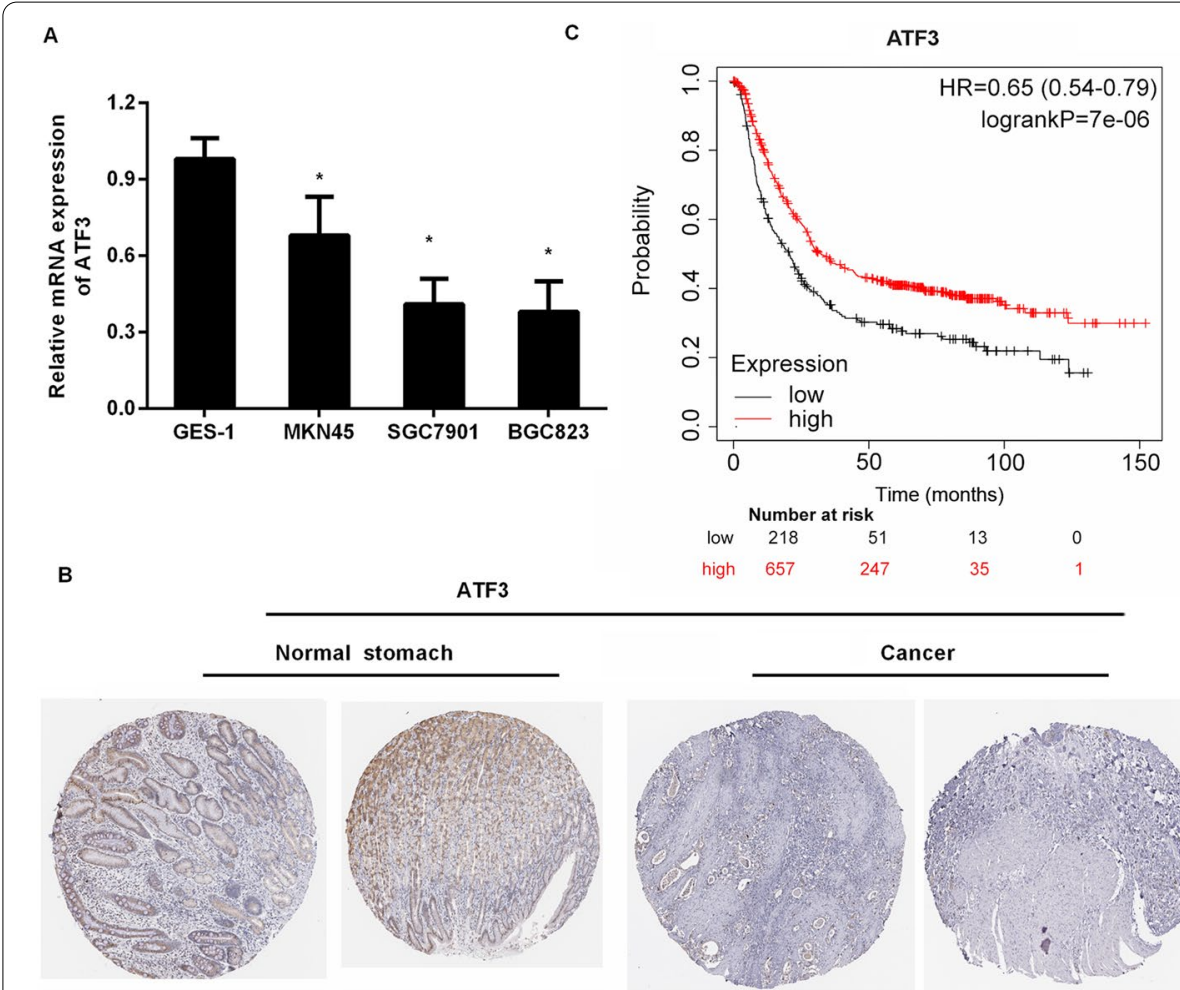

ATF3

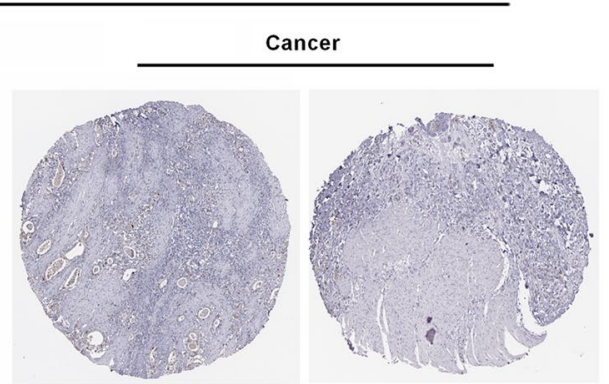

Fig. 1 ATF3 expression and prognosis in gastric cancer cells and tissues. A Transcription of ATF3 in human gastric epithelial cell GES-1 and GC cell lines (MNK45, SGC7901 and BGC823). B ATF3 protein expression in normal stomach and GC tissues. Images were obtained from the Human Protein Atlas online database. $\mathbf{C}$ Kaplan-Meier plotter analysis evaluated ATF3 expression and overall survival in GC patients. ${ }^{*} P<0.05$

cell GES-1, the mRNA levels of ATF3 were decreased in GC cell lines (MNK45, SGC7901 and BGC823). Lower transcription of ATF3 was observed in SGC7901 and BGC823 cells. Moreover, histochemistry data for GC using the Human Protein Atlas revealed that ATF3 protein was moderately expressed in normal gastric tissues, but weakly expressed in GC tissues (Fig. 1B). In Kaplan-Meier plotter analysis, GC patients with lower ATF3 expression had worse overall survival than those with higher ATF3 expression (Fig. 1C). These data indicate the critical roles of ATF3 in the progression of $\mathrm{GC}$.

\section{Down-regulation of ATF3 is validated in cisplatin-resistant gastric cancer cells}

As presented in Fig. 2A, cisplatin-resistant GC cells (SGC7901/DDP and BGC823/ $\mathrm{DDP})$ exhibited stronger resistance to cisplatin relative to the corresponding parental GC cells. Notably, compared with SGC7901 cells, lower transcription of ATF3 was observed in SGC7901/DDP cells (Fig. 2B). Moreover, an analogously lower ATF3 mRNA level was observed in BGC823/DDP cells relative to BGC823 cells (Fig. 2C). Similarly, the protein expression of ATF3 was decreased in SGC7901/DDP and BGC823/DDP, compared to their parental GC cells (Fig. 2D). 


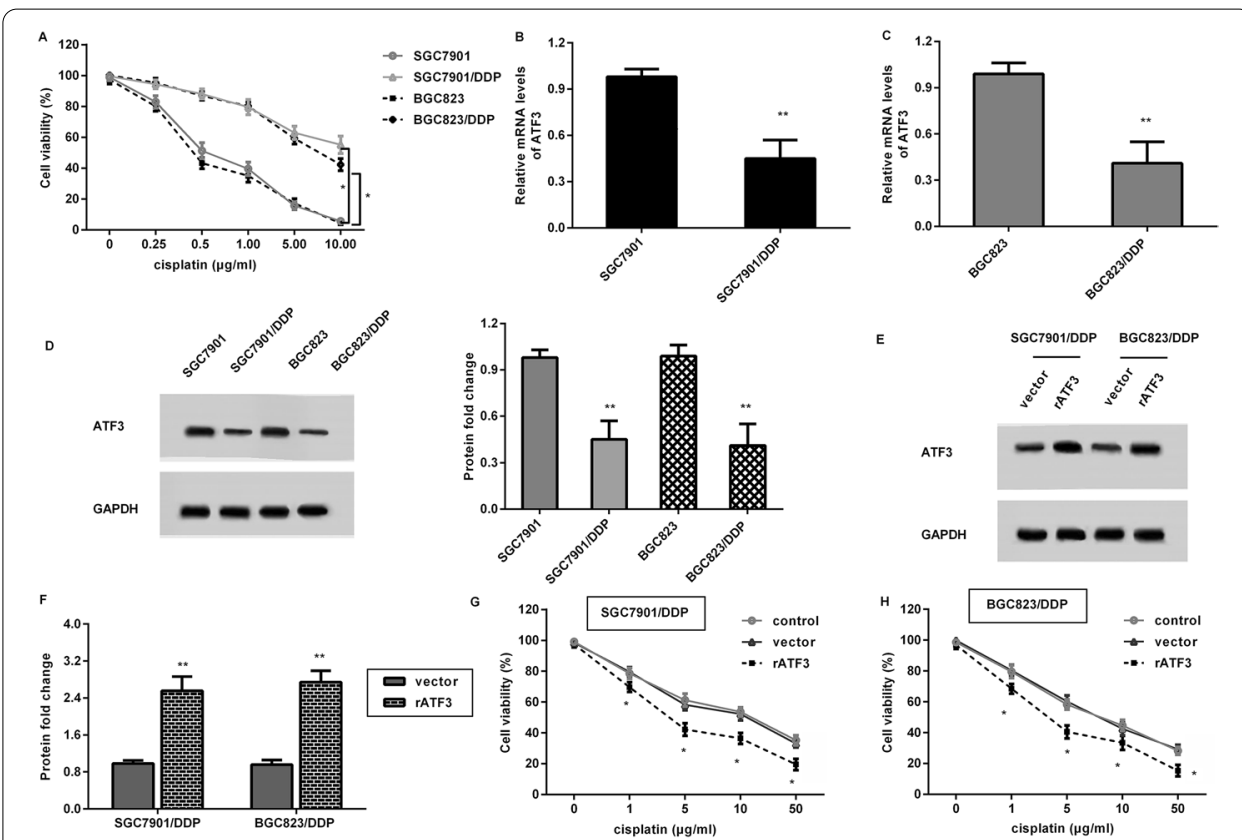

Fig. 2 Overexpression of ATF3 sensitized gastric cancer cells to cisplatin. A Cisplatin-resistant cells (SGC7901/ DDP and BGC823/DDP) and their parental cells (SGC7901 and BGC823 cells) were exposed to various doses of cisplatin. Then, a CCK-8 assay was performed to determine cell viability. B, C mRNA levels of ATF3 in cisplatin-resistant and parental GC cells. D Western blotting was conducted to analyze protein expression of ATF3. E, F SGC7901/DDP and BGC823/DDP cells were transfected with recombinant ATF3 plasmids, and the effect on ATF3 protein expression was then evaluated by western blotting. $\mathbf{G}, \mathbf{H}$ After transfection with rATF3 vectors, cell viability under cisplatin exposure was then detected. ${ }^{*} P<0.05 .{ }^{* *} P<0.01$

\section{ATF3 enhancement blunts gastric cancer cell resistance to cisplatin}

To further investigate the potential roles of ATF3 in cisplatin resistance in GC, SGC7901/DDP and BGC823/DDP were transfected with recombinant ATF3 plasmids (rATF3) to overexpress ATF3 (Fig. 2E and F). Intriguingly, up-regulation of ATF3 dramatically blunted SGC7901/DDP (Fig. 2G) and BGC823/DDP cell (Fig. 2H) resistance to cisplatin. These results reveal that ATF3 elevation may blunt GC cell resistance to cisplatin.

\section{Elevation of ATF3 affects ferroptosis in cisplatin-resistant gastric cancer cells}

Emerging evidence corroborates the critical function of ferroptosis in tumor chemotherapy resistance including GC $[10,25,26]$. Therefore, we further investigated the correlation between ATF3 and ferroptosis in GC cells. As shown in Fig. 3A, ATF3 overexpression further aggravated cisplatin-evoked cell death in SGC7901/DDP and BGC823/DDP. Additionally, lower ROS (Fig. 3B), MDA (Fig. 3C), and lipid peroxide (Fig. 3D) levels were detected in parental SGC7901 and BGC823 cells relative to SGC7901/DDP and BGC823/DDP cells, concomitant with higher GSH levels (Fig. 3E). These data imply that cisplatin-resistant GC cells exhibited lower ferroptosis. Of interest, overexpression of ATF3 enhanced ROS (Fig. 3F), MDA (Fig. 3G) 

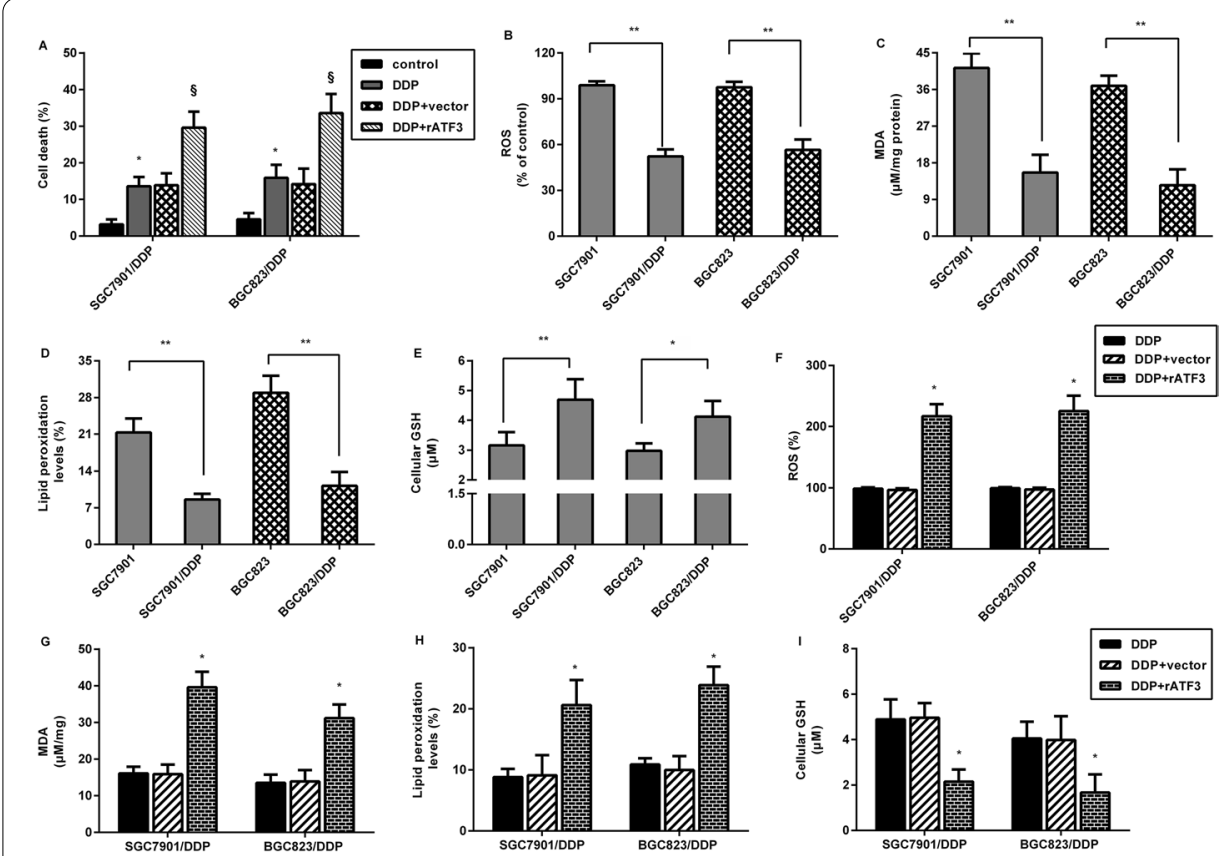

Fig. 3 ATF3 evoked ferroptosis in GC cells. A SGC7901/DDP and BGC823/DDP cells transfected with ATF3 plasmids were exposed to cisplatin. Cell death was then assessed by Annexin V-FITC/PI. B-E Ferroptosis was evaluated in cisplatin-resistant and parental GC cells by detecting ROS (B), MDA (C), lipid peroxidation (D) and GSH levels (E). F-I Cisplatin-resistant GC cells were treated with ATF3 vector transfection and cisplatin exposure, and the $\operatorname{ROS}(\mathbf{F}), \operatorname{MDA}(\mathbf{G})$, lipid peroxidation $(\mathbf{H})$ and GSH levels $(\mathbf{I})$ were then measured. ${ }^{*} P<0.05$. ${ }^{*} P<0.01$

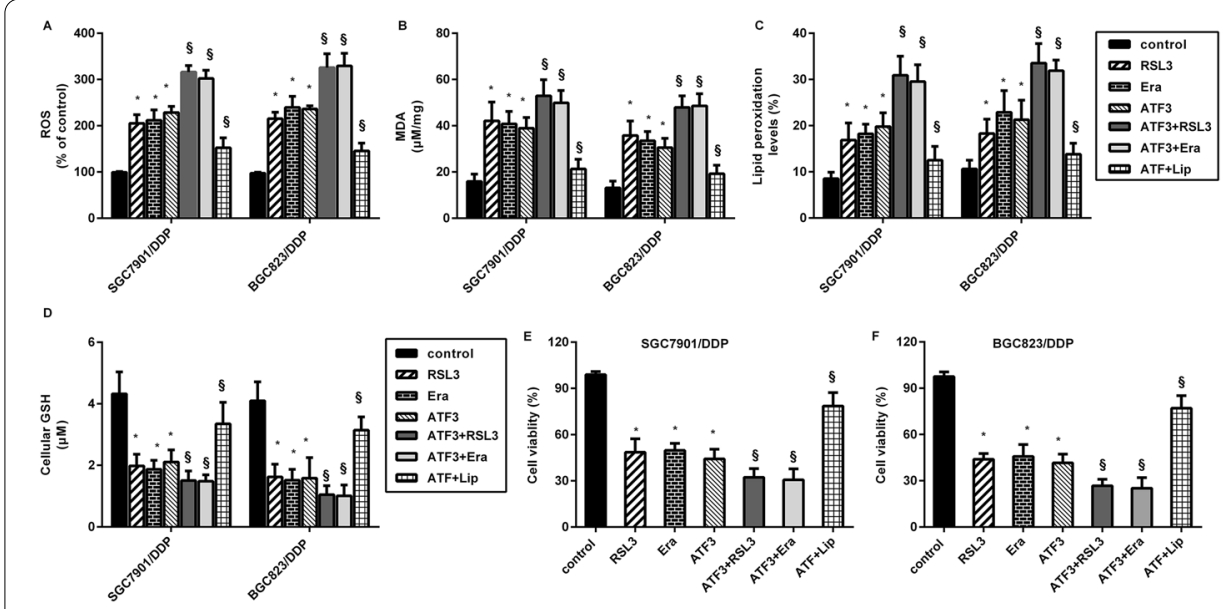

Fig. 4 Ferroptosis was responsible for the effects of ATF3 up-regulation on cisplatin resistance.

Cisplatin-resistant GC cells (SGC7901/DDP and BGC823/DDP) were treated with ATF3 transfection, ferroptosis agonist erastin, RSL3 and antagonist liproxstatin-1 under cisplatin conditions. Then, the levels of ROS (A), MDA (B), lipid peroxidation (C) and GSH (D) were determined. E, F Cell viability in SGC7901/DDP (E) and BGC823/ DD (F) was evaluated by CCK-8 assay. ${ }^{*} P<0.05$ vs. control group. ${ }^{\S} P<0.05$ vs. ATF3-treated group 


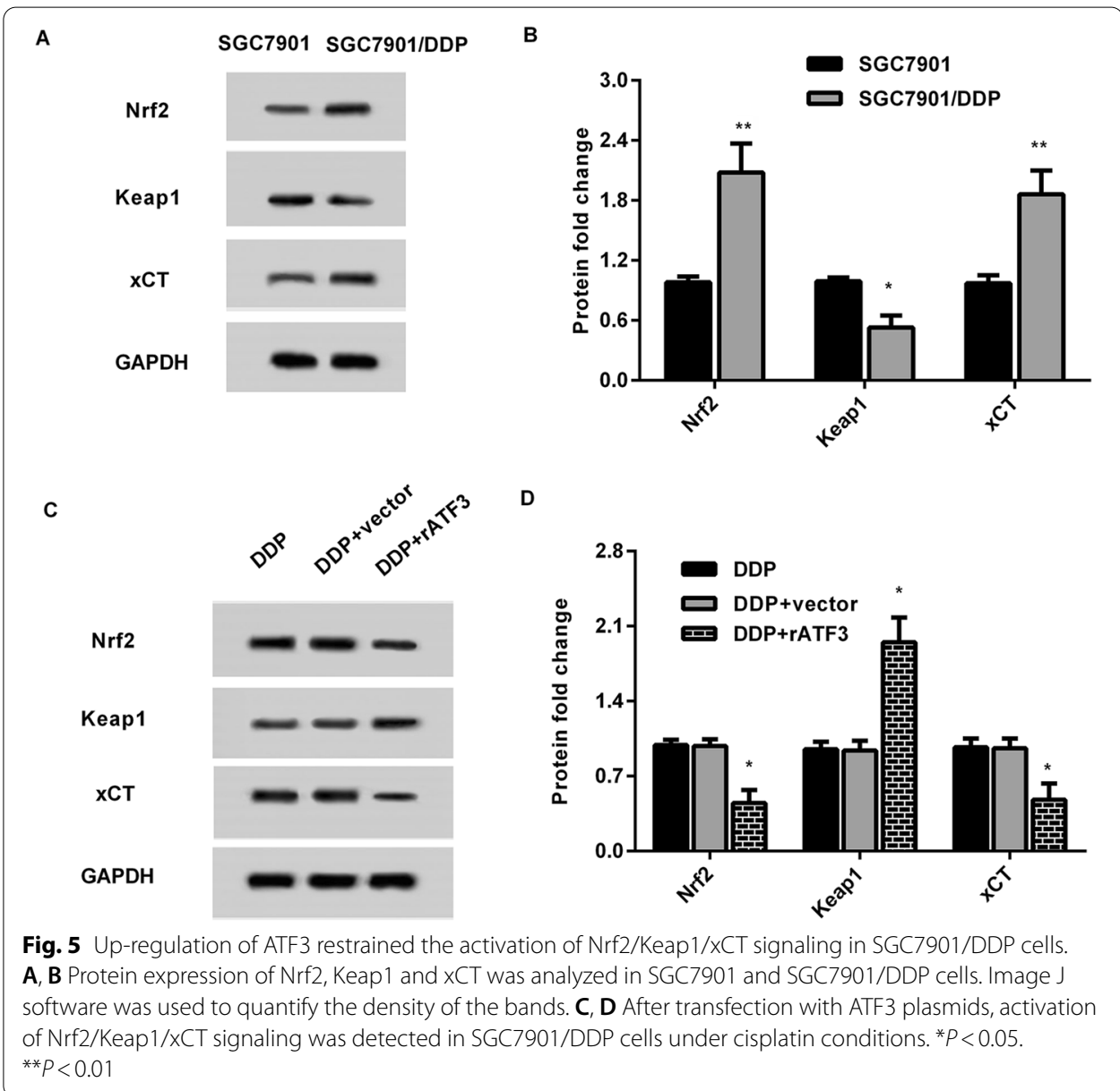

and lipid peroxidation (Fig. 3H) levels in SGC7901/DDP and BGC823/DDP cells. However, ATF3 enhancement inhibited GSH levels in SGC7901/DDP and BGC823/ DDP cells. These findings suggest that ATF3 up-regulation may evoke ferroptosis in cisplatin-resistant GC cells.

\section{Ferroptosis accounts for the effects of ATF3 up-regulation against cisplatin resistance}

To further elucidate the involvement of ferroptosis in ATF3-mediated cisplatin resistance in GC cells, the ferroptosis agonist erastin, RSL3 and the antagonist liproxstatin-1 were applied. As presented in Fig. 4A-C, activators of erastin and RSL3 (inhibition of GPx4 to induce ferroptosis) markedly enhanced ROS, MDA production and lipid peroxidation. Intriguingly, ATF3 overexpression also enhanced ROS production in SGC7901/ DDP and BGC823/DDP cells under cisplatin exposure; moreover, activation of ferroptosis by ATF3 overexpression together with the ferroptosis agonist erastin or RSL3 further elevated ROS levels. Nevertheless, the ferroptosis inhibitor liproxstatin-1 reversed ATF3-induced ROS production (Fig. 4A). Concomitantly, induction of ferroptosis further aggravated the effects of ATF3 on MDA (Fig. 4B), lipid peroxidation (Fig. 4C) and GSH levels (Fig. 4D); whilst suppression of ferroptosis by liproxstatin-1 overturned the above efficacy. Importantly, ATF3 elevation mitigated cell viability in cisplatin-treated 


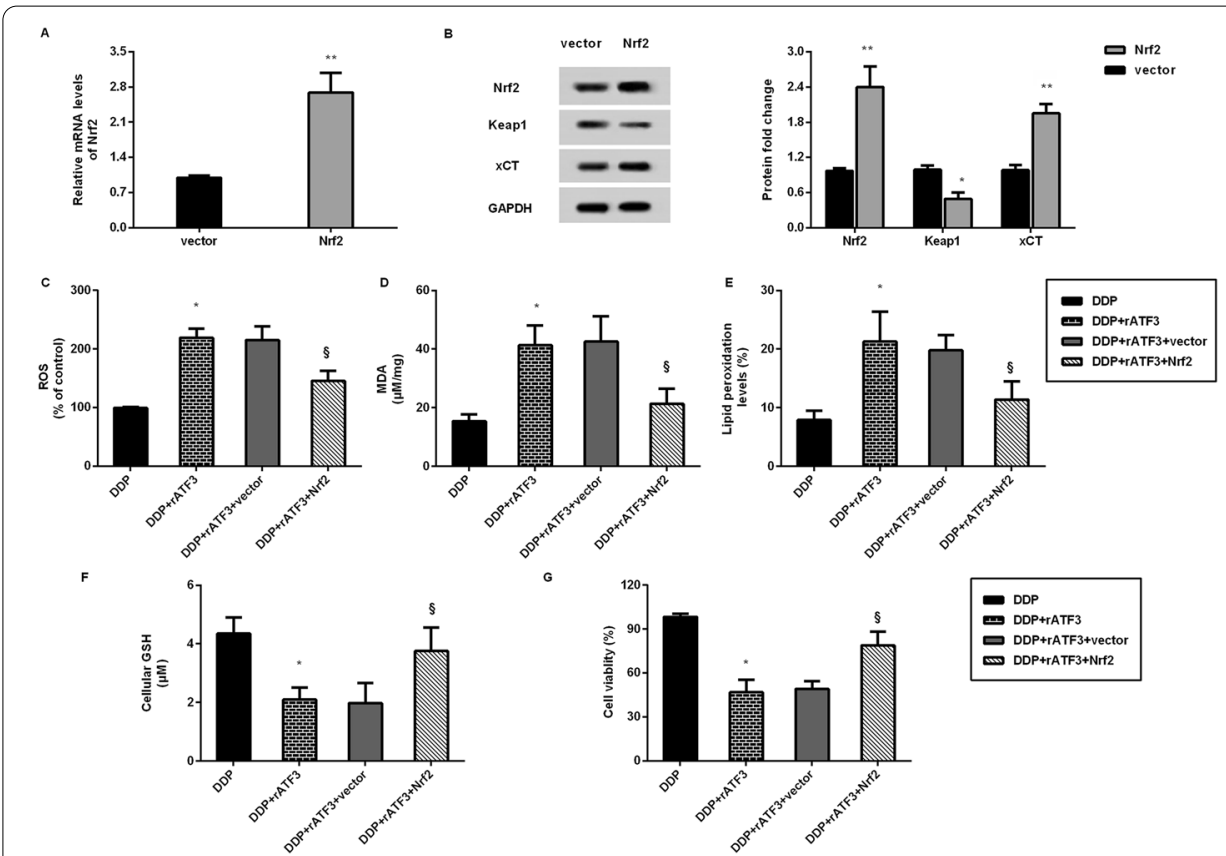

Fig. $6 \mathrm{Nrf2/Keap1/xCT}$ signaling involved in ATF3-mediated ferroptosis and cisplatin resistance in GC. A The effect of Nrf2 plasmid transfection on mRNA levels of Nrf2 was analyzed by qRT-PCR. B After transfection with Nrf2 vector, protein expression of Nrf2, Keap1 and $x C$ T was measured. ${ }^{*} P<0.05,{ }^{*} P<0.01$ vs. vector group. $\mathbf{C}$ SGC7901/DDP cells were treated with ATF3 plasmids and/or Nrf2 plasmids before cisplatin exposure. Then, the levels of ROS $(\mathbf{C}), \mathrm{MDA}(\mathbf{D})$, lipid peroxidation $(\mathbf{E}), \mathrm{GSH}(\mathbf{F})$ and cell viability $(\mathbf{G})$ were analyzed. ${ }^{*} P<0.05$ vs. DDP group. ${ }^{\S} P<0.05$ vs. DDP and ATF3 group

SGC7901/DDP (Fig. 4E) and BGC823/DDP cells (Fig. 4F), which was further reversed by liproxstatin-1. Moreover, ATF3 up-regulation further aggravated ferroptosis activator erastin- or RSL3-inhibited cell viability. These data indicate that ATF3 sensitizes GC cells to cisplatin via induction of ferroptosis.

\section{Activation of Nrf2/Keap1/xCT signaling in SGC7901/DDP is restrained by ATF3 overexpression}

Recent studies have established that Nrf2/Keap1 signaling has a key role in the development of cancers including GC [9, 27]. Intriguingly, higher protein expression of Nrf2 and xCT was observed in SGC7901/DDP cells relative to SGC7901 cells, concomitant with the decrease in Keap1 protein levels (Fig. 5A and B). Moreover, compared with DDP groups, overexpression of ATF3 restrained this signaling by decreasing Nrf2 and xCT expression and increasing Keap1 protein expression (Fig. 5C and D).

Nrf2/Keap1/xCT signaling is involved in ATF3-mediated ferroptosis and cisplatin resistance in gastric carcinoma

As shown in Fig. 6A, transfection with Nrf2 plasmids enhanced the transcription of Nr2 in SGC7901/DDP cells. Simultaneously, Nrf2 vector transfection increased protein expression of $\mathrm{Nrf} 2, \mathrm{xCT}$ and inhibited the Keap1 protein levels, indicating 
the restoration of Nrf2 signaling (Fig. 6B). Notably, ATF3 elevation enhanced ROS production (Fig. 6C), MDA levels (Fig. 6D) and lipid peroxidation (Fig. 6E), and decreased GSH levels (Fig. 6F). Nevertheless, reactivating Nrf2 signaling reversed the ATF3-mediated above effects (Fig. 6C-F), indicating that ATF3 may trigger ferroptosis by blocking the Nrf2/Keap1 pathway. Additionally, restoring the Nrf2 pathway increased cell viability in ATF3-overexpressed SGC7901/DDP cells (Fig. 6G).

\section{Discussion}

Gastric cancer (GC) represents a global health threat due to cancer-evoked death, and a majority of people with GC are usually diagnosed at an advanced stage. ATF3 has moved into the limelight within cancer research, where its expression is dysregulated in multiple cancer types; however, its expression and function are contradictory. Several studies corroborate obvious down-regulation and suppressive efficacy of ATF3 in carcinogenesis, including hepatocellular carcinoma [19], prostate cancer [18], and non-small cell lung carcinoma [20]. Conversely, ATF3 exhibits high expression levels in breast and skin carcinomas, and can act as an oncogene to participate in carcinogenesis $[15,16]$. In this study, we confirmed the decrease of ATF3 in GC cells relative to GES-1 human gastric epithelial cells. Importantly, histochemical data from the Human Protein Atlas revealed weak expression of ATF3 in GC tissues. Furthermore, Kaplan-Meier plotter analysis corroborated that ATF3 expression was positively related to the overall survival of GC patients. Therefore, these findings indicate the potential suppressive roles of ATF3 in GC.

Palliative chemotherapy is the mainstay for most patients with GC; nevertheless, poor or even no response to chemotherapy is often observed in the clinical treatment of GC patients due to acquired drug resistance that frequently results in a low 5-year survival rate [5]. In this study, we constructed cisplatin-resistant GC cells and substantiated the decrease of ATF3 in cisplatin-resistant cells relative to their parental cells, implying the potential of ATF3 in cisplatin resistance in GC. Importantly, ATF3 elevation sensitized cisplatin-resistant GC cells to cisplatin. Analogously with this finding, former research indicated that ATF3 accounts for the reinforcing effects on cisplatin cytotoxicity in nonsmall cell lung carcinoma [20]. Similarly, ATF3 promotes nasopharyngeal cancer cell sensitivity to paclitaxel [21]. Additionally, overexpression of ATF3 also enhances radioresistance of breast cancer [28].

We next corroborated one noteworthy finding that ATF3 elevation enhanced cell death in cisplatin-resistant GC cells. Concomitantly, cisplatin-resistant GC cells exhibited lower ROS, MDA, lipid peroxidation and higher GSH levels relative to parental cells, indicating the lower ferroptosis in cisplatin-resistant GC cells. Ferroptosis was recently defined as a newly discovered form of cell death that is a nexus between redox biology, metabolism, iron-dependent lipid peroxidation and health [29]. Unlike apoptosis, ferroptosis does not involve the activation of caspase. Ferroptosis can evoke ROS and MDA increase, and ultimately results in overwhelming lipid peroxidation to cause cell death [7]. Currently, fast-growing studies of ferroptosis in cancer have supported a novel viewpoint that induction of ferroptosis may ultimately aid in the discovery of novel therapeutic strategies to improve cancer treatment [25, 30]. For instance, ferroptosis triggered by tanshinone IIA or ferroptosis agonist erastin evokes GC cell death, indicating 
a new insight for GC intervention [30]. Recent research confirms that cisplatin acts as an inducer for ferroptosis, which opens up a new way for their utility in clinical practice [13]. Currently, triggering ferroptosis is recognized as a promising strategy to eradicate aggressive malignancies that are resistant to traditional chemotherapy [25]. Corroborating previous research $[11,24]$, we found that cisplatin-resistant GC cells exhibited lower ferroptosis. In this study, ATF3 elevation induced ferroptosis in cisplatin-resistant GC cells. Notably, a recent study confirmed the pro-ferroptosis efficacy of ATF3 in fibrosarcoma cells [22]. Importantly, ATF3 elevation evoked ferroptosis and ultimately sensitized cisplatin-resistant cells to cisplatin, whilst the antagonist liproxstatin-1 overturned ATF3 effects on ferroptosis and subsequent cisplatin resistance. These findings highlight that ATF3 may blunt cisplatin-resistant GC cells to cisplatin by evoking ferroptosis. Analogously, ferroptosis suppression promotes chemoresistance in GC [26].

Mechanistically, the activation of Nrf2/Keap1/xCT signaling was observed in cisplatin-resistant GC cells; however, ATF3 elevation restrained this activation. Nrf2 is a cytosolic transcription factor that can bind and suppress Keap1 expression to regulate antioxidant and stress-related events. In normal conditions, activation of the Nrf2/ Keap1 pathway induces cytoprotection and antagonizes tissue injury [31,32]. Over the past few years, abundant research has substantiated tumor suppressor efficacy of Nrf2 in carcinogenesis [27]. Contradictorily, inhibition of Nrf2 activation has recently been considered as a promising approach to suppress tumor growth and overcome chemoresistance [27]. Intriguingly, recent research has implicated Nrf2 signaling in restraining lipid peroxidation and ferroptosis $[9,33]$. In the current study, reactivating Nrf2/Keap1/xCT signaling overturned ATF3-mediated suppression in ferroptosis and subsequent cisplatin resistance in resistant GC cells. Suppression of xCT usually leads to GSH depletion and finally results in ferroptosis in cancer cells [22, 25]. Notably, activation of the Nrf2/Keap1 pathway increases xCT (SLC7A11) expression and diminishes ferroptosis, thus facilitating glioma cell growth [9]. Especially, blockage of the Nrf2 pathway overcomes resistance of cisplatin-resistant head and neck cancer cells to cisplatin by inducing ferroptosis[24]. Thus, these observations prompt us to conclude that Nrf2/Keap1/xCT-mediated ferroptosis may account for the efficacy of ATF3 against cisplatin resistance in GC cells. As expected, restoring the Nrf2 signaling muted ATF3-mediated ferroptosis and cisplatin resistance in GC cells. Notably, in this study, we confirmed the correlation between ATF3 and NRf2 in ferroptosis inhibition in GC. However, what is the potential mechanisms by which ATF3 blocks NRF2? A recent study confirmed that ATF3-mediated suppression is a consequence of direct ATF3-Nrf2 protein-protein interactions [34]. Therefore, does ATF3 directly affect Nrf2 expression by protein-protein interaction, or indirectly regulate Nrf2 expression by other common pathways? All of these questions are still undefined and should be explored in our future research.

\section{Conclusions}

Collectively, one noteworthy observation in the current study was that expression of ATF3 was decreased in GC cells and tissues, and was positively related to the overall survival of GC patients. Notably, lower expression was observed in cisplatin-resistant 
cells relative to their parental cells. Especially, the current study highlighted a novel finding that elevation of ATF3 sensitized cisplatin-resistant GC cells to cisplatin by evoking ferroptosis via inhibition of $\mathrm{Nrf} 2 / \mathrm{Keap} 1 / \mathrm{xCT}$ signaling. Consequently, the present data may support a promising therapeutic approach to overcome cisplatin resistance in the treatment of GC patients.

\section{Abbreviations}

DDP: Cisplatin; GC: Gastric carcinoma; ATF3: Activating transcription factor 3; ROS: Reactive oxygen species; MDA: Malondialdehyde; I/R: Ischemia-reperfusion; Nrf2: Nuclear factor (erythroid-derived)-like 2; GSH: Glutathione.

\section{Acknowledgements}

None.

\section{Authors' contributions}

DZF designed the concept, and conducted the experiments. DZF and CXW performed biological informatics, cell culture and cisplatin resistance analysis. LY and RY carried out the mRNA, western blotting and ferroptosis analysis. All authors read and approved the final manuscript.

\section{Funding}

This research did not receive any specific grant from funding agencies in the public, commercial, or not-for-profit sectors.

Availability of data and material

All data generated or analyzed during this study are included in this published article.

\section{Declarations}

Ethics approval and consent to participate

Not applicable.

\section{Consent to participate}

Not applicable.

\section{Competing interests}

The authors declare that they have no competing interests.

\section{Author details}

${ }^{1}$ Department of General Surgery, First Affiliated Hospital of China Medical University, Liaoning Province Shenyang City Heping District Nanjingbei Road 155, Shenyang 110001, Liaoning, People's Republic of China. ${ }^{2}$ Department of General Surgery, Liaoning Health Industry Group, Benxi Iron \& Steel Group, General Hospital, Benxi 117000, Liaoning, People's Republic of China.

Received: 11 March 2021 Accepted: 25 May 2021

Published online: 07 June 2021

\section{References}

1. Smyth EC, Nilsson M, Grabsch HI, van Grieken NC, Lordick F. Gastric cancer. Lancet. 2020;396:635-48.

2. Venerito M, Vasapolli R, Rokkas T, Malfertheiner P. Gastric cancer: epidemiology, prevention, and therapy. Helicobacter. 2018;23:e12518.

3. Petryszyn P, Chapelle N, Matysiak-Budnik T. Gastric Cancer: Where Are We Heading? Dig Dis. 2020;38:280-5.

4. Bray F, Ferlay J, Soerjomataram I, Siegel RL, Torre LA, Jemal A. Global cancer statistics 2018: GLOBOCAN estimates of incidence and mortality worldwide for 36 cancers in 185 countries. CA Cancer J Clin. 2018;68:394-424.

5. Zhao TT, Xu H, Xu HM, Wang ZN, Xu YY, Song YX, et al. The efficacy and safety of targeted therapy with or without chemotherapy in advanced gastric cancer treatment: a network meta-analysis of well-designed randomized controlled trials. Gastric Cancer. 2018;21:361-71.

6. Dixon SJ, Lemberg KM, Lamprecht MR, Skouta R, Zaitsev EM, Gleason CE, et al. Ferroptosis: an iron-dependent form of nonapoptotic cell death. Cell. 2012;149:1060-72.

7. Xie Y, Hou W, Song X, Yu Y, Huang J, Sun X, et al. Ferroptosis: process and function. Cell Death Differ. 2016;23:369-79.

8. Liu P, Feng Y, Li H, Chen X, Wang G, Xu S, et al. Ferrostatin-1 alleviates lipopolysaccharide-induced acute lung injury via inhibiting ferroptosis. Cell Mol Biol Lett. 2020;25:10.

9. Fan Z, Wirth AK, Chen D, Wruck CJ, Rauh M, Buchfelder M, et al. Nrf2-Keap1 pathway promotes cell proliferation and diminishes ferroptosis. Oncogenesis. 2017;6:e371.

10. Roh JL, Kim EH, Jang HJ, Park JY, Shin D. Induction of ferroptotic cell death for overcoming cisplatin resistance of head and neck cancer. Cancer Lett. 2016;381:96-103. 
11. Liu Q, Wang K. The induction of ferroptosis by impairing STAT3/Nrf2/GPx4 signaling enhances the sensitivity of osteosarcoma cells to cisplatin. Cell Biol Int. 2019;43:1245-56.

12. Lou JS, Zhao LP, Huang ZH, Chen XY, Xu JT, Tai WC, et al. Ginkgetin derived from Ginkgo biloba leaves enhances the therapeutic effect of cisplatin via ferroptosis-mediated disruption of the Nrf2/HO-1 axis in EGFR wild-type non-small-cell lung cancer. Phytomedicine. 2021;80:153370.

13. Guo J, Xu B, Han Q, Zhou H, Xia Y, Gong C, et al. Ferroptosis: a novel anti-tumor action for cisplatin. Cancer Res Treat. 2018;50:445-60.

14. Ku HC, Cheng CF. Master regulator activating transcription factor 3 (ATF3) in metabolic homeostasis and cancer. Front Endocrinol (Lausanne). 2020;11:556.

15. Kim S, Song NJ, Bahn G, Chang SH, Yun UJ, Ku JM, et al. Atf3 induction is a therapeutic target for obesity and metabolic diseases. Biochem Biophys Res Commun. 2018;504:903-8.

16. Yin X, Wolford CC, Chang YS, McConoughey SJ, Ramsey SA, Aderem A, et al. ATF3, an adaptive-response gene, enhances TGF $\{$ beta\} signaling and cancer-initiating cell features in breast cancer cells. J Cell Sci. 2010;123:3558-65.

17. Hao ZF, Ao JH, Zhang J, Su YM, Yang RY. ATF3 activates Stat3 phosphorylation through inhibition of p53 expression in skin cancer cells. Asian Pac J Cancer Prev. 2013;14:7439-44.

18. Wang Z, Xu D, Ding HF, Kim J, Zhang J, Hai T, et al. Loss of ATF3 promotes Akt activation and prostate cancer development in a Pten knockout mouse model. Oncogene. 2015;34:4975-84.

19. Chen C, Ge C, Liu Z, Li L, Zhao F, Tian H, et al. ATF3 inhibits the tumorigenesis and progression of hepatocellular carcinoma cells via upregulation of CYR61 expression. J Exp Clin Cancer Res. 2018;37:263.

20. Bar J, Hasim MS, Baghai T, Niknejad N, Perkins TJ, Stewart DJ, et al. Induction of activating transcription factor 3 is associated with cisplatin responsiveness in non-small cell lung carcinoma cells. Neoplasia. 2016;18:525-35.

21. Zhu Y, He D, Bo H, Liu Z, Xiao M, Xiang L, et al. The MRVI1-AS1/ATF3 signaling loop sensitizes nasopharyngeal cancer cells to paclitaxel by regulating the Hippo-TAZ pathway. Oncogene. 2019;38:6065-81.

22. Xu F, Ye Z, Tao S, Liu W, Su J, Fang X, et al. Ligustilide alleviates podocyte injury via suppressing the SIRT1/NFkappaB signaling pathways in rats with diabetic nephropathy. Ann Transl Med. 2020;8:1154.

23. Wang Q, Chen Q, Zhu L, Chen M, Xu W, Panday S, et al. JWA regulates TRAlL-induced apoptosis via MARCH8mediated DR4 ubiquitination in cisplatin-resistant gastric cancer cells. Oncogenesis. 2017;6:e353.

24. Shin D, Kim EH, Lee J, Roh JL. Nrf2 inhibition reverses resistance to GPX4 inhibitor-induced ferroptosis in head and neck cancer. Free Radic Biol Med. 2018;129:454-62.

25. Liang C, Zhang X, Yang M, Dong X. Recent progress in ferroptosis inducers for cancer therapy. Adv Mater. 2019;31:e1904197.

26. Zhang H, Deng T, Liu R, Ning T, Yang H, Liu D, et al. CAF secreted miR-522 suppresses ferroptosis and promotes acquired chemo-resistance in gastric cancer. Mol Cancer. 2020;19:43.

27. Jeddi F, Soozangar N, Sadeghi MR, Somi MH, Samadi N. Contradictory roles of Nrf2/Keap1 signaling pathway in cancer prevention/promotion and chemoresistance. DNA Repair (Amst). 2017;54:13-21.

28. Zhao W, Sun M, Li S, Chen Z, Geng D. Transcription factor ATF3 mediates the radioresistance of breast cancer. J Cell Mol Med. 2018;22:4664-75.

29. Stockwell BR, Friedmann Angeli JP, Bayir H, Bush Al, Conrad M, Dixon SJ, et al. Ferroptosis: a regulated cell death nexus linking metabolism, redox biology, and disease. Cell. 2017;171:273-85.

30. Guan Z, Chen J, Li X, Dong N. Tanshinone IIA induces ferroptosis in gastric cancer cells through p53-mediated SLC7A11 down-regulation. Biosci Rep. 2020;40:8.

31. Shen Y, Liu X, Shi J, Wu X. Involvement of Nrf2 in myocardial ischemia and reperfusion injury. Int J Biol Macromol. 2019;125:496-502.

32. Wang W, Yang X, Chen Q, Guo M, Liu S, Liu J, et al. Sinomenine attenuates septic-associated lung injury through the Nrf2-Keap1 and autophagy. J Pharm Pharmacol. 2020;72:259-70.

33. Dodson M, Castro-Portuguez R, Zhang DD. NRF2 plays a critical role in mitigating lipid peroxidation and ferroptosis. Redox Biol. 2019;23:101107.

34. Brown SL, Sekhar KR, Rachakonda G, Sasi S, Freeman ML. Activating transcription factor 3 is a novel repressor of the nuclear factor erythroid-derived 2-related factor 2 (Nrf2)-regulated stress pathway. Cancer Res. 2008;68:364-8.

\section{Publisher's Note}

Springer Nature remains neutral with regard to jurisdictional claims in published maps and institutional affiliations.

Ready to submit your research? Choose BMC and benefit from:

- fast, convenient online submission

- thorough peer review by experienced researchers in your field

- rapid publication on acceptance

- support for research data, including large and complex data types

- gold Open Access which fosters wider collaboration and increased citations

- maximum visibility for your research: over $100 \mathrm{M}$ website views per year

At BMC, research is always in progress.

Learn more biomedcentral.com/submissions 